Pacific Journal of Mathematics

SPECTRAL SYNTHESIS IN PRODUCTS AND QUOTIENTS OF 


\title{
SPECTRAL SYNTHESIS IN PRODUCTS AND QUOTIENTS OF HYPERGROUPS
}

\author{
AJAY KUMAR AND AJIT IQBAL SINGH*
}

In this paper we first discuss the coset spaces $K / H$ and $K / / H$ of left cosets and double cosets respectively of a hypergroup $K$ by a compact subhypergroup $H$. This development is then used to obtain some results connecting spectral synthesis for $L^{1}(K / H)$ to that for $L^{1}(K)$ when $K$ is commutative. We also indicate that some of the results for quotient group carry over to $K / H$ when $H$ is a subgroup of the center $Z(K)$ of $K$. Finally we discuss how Malliavin's theorem fails in a strong way in many hypergroups and further show that for certain closed sets of the form $E_{1} \times E_{2}$ in $\hat{K}_{1} \times \hat{K}_{2}$, where $K_{1}=\boldsymbol{R}^{+}, Z^{+}$ etc. and $K_{2}$ is a locally compact commutative hypergroup such that the dual $\hat{K}_{2}$ of $K_{2}$ is a $\sigma$-compact hypergroup, $E_{1} \times E_{2}$ can inherit various properties of $E_{1}$ such as being nonspectral, non ultra-strong Ditkin for the respective hypergroup algebras.

1. Introduction. The propose of this paper is to discuss spectral synthesis for quotients and products of hypergroups. The basic development of harmonic analysis for hypergroups can be found in ([10], [11], [13], [20] and [21]). Spectral synthesis for hypergroups has been developed in ([9], [7] and [8]). The motivation for all this has been the fact that hypergroups arise in a natural way as a double coset space, the space of conjugacy classes of a compact group and harmonic analysis on them is closely related to that of the groups, a survey of this can be found in ([18] and [6]). Our main reference for the basic theory of hypergroups will be [13] and most of the further notation and terminology is as in ([12], [9] and [7]). Throughout this paper $K$ will denote a locally compact hypergroup (same as 'convo' in [13]) possessing a Haar measure $m, L^{1}(K)=L^{1}(m)$ the convolution algebra and $H$ a compact subhypergroup of $K$. In $\S 2$ we discuss the quotient hypergroup $K / / H$ of double cosets and the Weil's formula for $L^{1}(K / / H)$, also we briefly describe the situation for the left coset space $K / H$ as well. In the next section we confine our attention to those commutative hypergroups $K$ for which the dual $\hat{K}$ is a hypergroup under pointwise operations. We first identify the dual of $K / H$ as the subhypergroup $H^{\perp}$ of $\hat{K}$ and relate spectral synthesis of $L^{1}(K / H)$ with that of $L^{1}(K)$. In particular, we show that $\Delta \subset H^{\perp}$ is spectral for $L^{1}(K / H)$ if it is so for $L^{1}(K)$ and $\Delta$ is strong Ditkin for $L^{1}(K / H)$ if and only if it is so for $L^{1}(K)$. In $\S 4$ we again take $K$ to be a commutative hypergroup

\footnotetext{
* née A jit Kaur Chilana.
} 
whose dual $\hat{K}$ is a hypergroup and prove similar results for the coset space $K / H$, when $H$ is a subgroup of the center $Z(K)$ of $K$ [19]. In the last section we discuss spectral synthesis for the hypergroup algebra $L^{1}\left(K_{1} \times K_{2}\right)$, where $K_{1}$ can be one of the $\boldsymbol{R}^{+}, Z^{+}$, Naimark example ([13], 9.5) and the dual $\hat{F}$ of the hypergroup $F$ of conjugacy classes of the compact group $S U(2)$ ([13], 15.4 and [9], 4.7) and $K_{2}$ is any commutative hypergroup whose dual $\hat{K}_{2}$ is a $\sigma$-compact hypergroup under pointwise operations. In particular we obtain methods to find various nonspectral sets, non ultra-strong Ditkin sets in $\hat{K}_{1} \times \hat{K}_{2}$. In this process we also show how Malliavin's theorem fails in a strong way in many hypergroups. We just remark that the analogues for Segal algebras of some of the results on spectral synthesis in this paper can be easily formulated and proved based on [7]. ${ }^{1}$

2. Quotient hypergroups. In this section $K$ will be a locally compact hypergroup with Haar measure $m$ and $H$ a compact subhypergroup of $K$ with normalized Haar measure $\sigma$. As shown in ([13], §14) the quotient map $\pi$ on $K$ onto the double coset space $K / / H=\{H * x * H: x \in K\}$ gives rise to maps $\pi_{*}$, on $M(K)$ to $M(K / / H)$ and $\pi^{*}$ on $M(K / / H)$ to $M(K)$ defined by

$$
\pi_{*}(\mu)=\mu \circ \pi^{-1} \text { and } \pi^{*}\left(p_{H^{*} x_{H}}\right)=\sigma * p_{x} * \sigma .
$$

Further $K / / H$ can be made into a hypergroup with convolution defined by

$$
\begin{aligned}
\int_{K / / H} f d p_{H^{*} x^{*} H} * p_{H^{*} y^{*} H} & =\int_{K / / H} f d \pi_{*}\left(\pi^{*}\left(P_{H^{*} x^{*} H}\right) * \pi^{*}\left(p_{H^{*} y^{*} H}\right)\right) \\
& =\int_{K} f \circ \pi d p_{x} * \sigma * p_{y}
\end{aligned}
$$

for all $f \in B^{\infty}(K / / H)$. A Haar measure on $K / / H$ is given by $\dot{m}=$ $\int_{K} p_{H^{*} x^{*} H} m(d x)$. Since the mapping $\dot{x}=H * x * H \rightarrow \sigma * p_{x} * \sigma$ is the recomposition of $\pi$ consistent with $m([13], 14.2 \mathrm{H})$, we have the following result.

THeORem 2.1. (Weil's formula) The Haar measure $\dot{m}$ on $K / / H$ can be so chosen that

$$
\int_{K / / H} \int_{H}(\sigma * f)(x * \xi) d \sigma(\xi) d \dot{m}(\dot{x})=\int_{K} f d m, \text { for } f \text { in } L^{1}(K)
$$

or equivalently

$$
\int_{K / / H} \int_{K} f d \sigma * p_{x} * \sigma d \dot{m}(\dot{x})=\int_{K} f d m .
$$

\footnotetext{
${ }^{1}$ The present paper along with [7] and [8] forms a major part of [23].
} 
REMARK 2.2. The reason why we call the above theorem the Weil's formula is that in case $H$ is strongly normal in the sense that $p_{x} * \sigma=\sigma * p_{x}$ for all $x$ in $K$ we have that $x * H=H * x=H * x * H$ for each $x$ in $K$ and (A) takes the form

$$
\int_{K / H} \int_{H} f(x * \xi) d \sigma(\xi) d \dot{m}(\dot{x})=\int_{K} f(x) d m(x)
$$

which is the natural analogue of Weil's formula in the group case.

Lemma 2.3. Let $F_{1}$ be a compact subset of $K / / H$ then there exists a compact subset $F_{2}$ of $K$ such that $\pi\left(F_{2}\right)=F_{1}$.

Proof. Follows from ([13], 13.2A).

THEOREM 2.4. Let $T_{I I}$ be the mapping defined by $T_{I I} f(H * x * H)=$ $\int_{K} f d \sigma * p_{x} * \sigma, f \in L^{1}(K)$. Then

(i ) for $g \in L^{1}(K / / H)$ we have (a) $f=g \circ \pi \in L^{1}(K)$.

(b) $f(\eta * x * \xi)=f(x)$ for all $\eta, \xi \in H$ and $x \in K$.

(c) $\int_{K / / H} g(\dot{x}) d \dot{m}(\dot{x})=\int_{K} g \circ \pi(x) d m(x)$.

(ii) $T_{H}$ is a bounded linear map of $L^{1}(K)$ onto $L^{1}(K / / H)$ with norm 1.

(iii) $T_{H}$ induces the isometric isomorphism $\pi^{*-1}$ on the subspace of $H$-invariant functions in $L^{1}(K)$ onto $L^{1}(K / / H)$.

(iv) In case $H$ is strongly normal, $T_{H}$ is an algebra homomorphism.

Proof. For $g \in L^{1}(K / / H), g \circ \pi$ is Borel measurable ([13], 13.2G). Using Theorem 2.1 simple computations show that $g \circ \pi \in L^{1}(K)$. Also $g \circ \pi(\eta * x * \xi)=g \circ \pi(x)$ for all $x \in K$ and $\eta, \xi \in H$ and $T_{H}(g \circ \pi)=g$ with $\|g \circ \pi\|_{1}=\|g\|_{1}$. Thus we have (i) (a), (b) and (iii) and (i)(c) follows from $([13], 13.2 \mathrm{H})$. (ii) can be proved using Theorem 2.1. For (iv) let us assume that $p_{x} * \sigma=\sigma * p_{x}$ for all $x \in K$. Simple computations show that $\left(T_{H} f\right)_{\dot{x}}=T_{H} f_{x}$ for all $x \in K$, further this fact can be used to prove that $T_{I I}$ is an algebra homomorphism using ([13], 5.1D) and the fact that $(f * g)_{x}=f_{x} * g$.

REMARK 2.5. Mackey [14] and Bruhat [5] generalized Weil's formula for quotient space of a locally compact group $G$ by a closed subgroup $H$ (not necessarily normal) which is also discussed as the formula of Mackey-Bruhat for quasi-invariant measures $m$ ([16], VIII). In case $H$ is compact the quotient space $G / H$ can also be thought of as the semi-convo of cosets with convolution given by 
$p_{x^{*} H} p_{y^{*} H}=\int_{H} p_{x t y H} d \sigma(t)$, where $\sigma$ is the Haar measure of $H$. It can be shown that the measure $\dot{m}$ in ([16], VIII, 1.1) is left invariant and therefore is the Haar measure on $G / H([13], 5 \cdot 2,8.2)$. Thus Weil's formula of Mackey and Bruhat fits well as Weil's formula in the hypergroup setting.

Now if $K$ is a hypergroup with Haar measure $m$ and $\pi_{I I}$ is the quotient map on $K$ onto $K / H$, then we can show that $K / H$ is a semi-convo (using $2.3 \mathrm{H}, 10.3 \mathrm{~B}, 13.2 \mathrm{~A}, \mathrm{~B}, \mathrm{C}$ and analogues of $14.1 \mathrm{~A}$, 14.1D of [13]) with Haar measure $\dot{m}=\int_{K} p_{x^{*} H} m(d x)$. Since $H$ is compact using $5.3 \mathrm{C}$ of [13] we have that $\Delta_{I I}(H)$ and $\Delta_{K}(H)$ are compact subgroups of the multiplicative group of positive real numbers and therefore $\Delta_{I I}(H)=\{1\}=\Delta_{K}(H)$. This helps us in showing that the map $x * H \rightarrow p_{x} * \sigma$ is a recomposition of $\pi_{H}$ consistent with $m$ or equivalently we have the Weil's formula in the following form

$$
\int_{/ H K} \int_{H} f(x * \xi) d \sigma(\xi) d \dot{m}(\dot{x})=\int_{K} f d m .
$$

3. Spectral synthesis for $L^{1}(K / H)$. In this section we consider only those $K$ which are commutative and for which $\hat{K}$ is a hypergroup with pointwise operations. Then every compact subhypergroup $H$ of $K$ is strongly normal and thus gives rise to the quotient hypergroup $K / H$ which is commutative and therefore has a Haar measure by ([21] or [13], 14.2). As in $\S 2$ above the Haar measure $\dot{m}$ of $K / H$ can be so chosen as to satisfy Weil's formula. In this case the convolution algebra $L^{1}(K / H)$ is commutative and we can discuss spectral synthesis for this algebra. For the sake of uniformity we further assume that $\hat{K}=\mathfrak{X}_{b}(K)$, eventhough for some of the results it can be replaced by weaker conditions such as the regularity of $L^{1}(K)$ on $\mathfrak{X}_{b}(K)$ ([9], [7]).

Lemma 3.1. Let $x, y \in K, x * H=y * H$ implies that

$$
\int_{K} f d p_{x} * \sigma=\int_{K} f d p_{y} * \sigma \text { for all } f \in C_{b}(K) .
$$

Proof. By ([13], 3.2G), $\int_{K} f d p_{x} * \sigma=\int_{x^{* I I}} f d p_{x} * \sigma$ and now modify 14.1B of [13] using compactness of $(x * H) \cup(y * H)$ and ([9], 2.5).

THEOREM 3.2. For $\varphi$ in $H^{\perp}, \varphi^{\prime}(x * H)=\varphi(x)$ defines an element of $(K / H)^{\wedge}, \varphi \rightarrow \varphi^{\prime}$ is a one-to-one map of $H^{\perp}$ onto $(K / H)^{\wedge}$.

Proof. We first note a fact often used in the proof namely for 
$\varphi$ in $H^{\perp}$

$$
\int_{K} \varphi d \sigma=\int_{I I} \varphi d \sigma=1
$$

Let $x \in K$ and $y \in x * H$. Then $([13], 10.3 \mathrm{~A})$ gives $x * H=y * H . \quad$ By Lemma $3.1 \int_{K} \varphi d p_{x} * \sigma=\int_{K} \varphi d p_{y} * \sigma$, so using ([13], 3.1E) and the homomorphism property of $\varphi$, we have that $\varphi(x)=\varphi(y)$. Hence $\varphi^{\prime}$ is well defined. Now using ([13], 4.2H) at appropriate places it is easy to show that $\phi^{\prime}((x * H) *(y * H))=\varphi^{\prime}(x * H) \varphi^{\prime}(y * H)$. Clearly $\phi^{\prime}$ is hermitian and $([13], 10.3 \mathrm{~B})$ implies that $\varphi^{\prime}$ is continuous and therefore $\varphi^{\prime} \in(K / H)^{\wedge}$. Obviously $\varphi \rightarrow \varphi^{\prime}$ is a well defined map. Using ([13], 3.1E, 10.3B) again we have that if $r \varepsilon(K / H)^{\wedge}$ then $\varphi(x)=$ $\dot{\gamma}(x * H)$ defines an element in $H^{\perp}$ such that $\varphi^{\prime}=\dot{\psi}$. Thus $\eta$ given by $\eta(\varphi)=\phi^{\prime}$ maps $H^{\perp}$ onto $(K / H)^{\wedge}$.

If $F_{1}$ is a compact subset of $K / H$ then by Lemma 2.3 there exists a compact subset $F_{2}$ of $K$ such that $\pi\left(F_{2}\right)=F_{1}$. Consider $W\left(F_{1}, \chi, \varepsilon\right)=\left\{\varphi \in(K / H)^{\wedge}:|\chi(x * H)-\varphi(x * H)|<\varepsilon\right.$ for all $\left.x * H \in F_{1}\right\}$ and $W\left(F_{2}, \chi^{\prime}, \varepsilon\right)=\left\{\gamma \in \hat{K}:\left|\chi^{\prime}(x)-\gamma(x)\right|<\varepsilon\right.$ for all $\left.x \in F_{2}\right\} \cap H^{\lrcorner}$. Clearly $\eta\left(W\left(F_{2}, \chi^{\prime}, \varepsilon\right)\right)=W\left(F_{1}, \chi, \varepsilon\right)$ and hence the result.

COROLlARY 3.3. $(K / H)^{\wedge}$ is a commutative hypergroup under pointwise operations.

Corollary 3.4. For each $x \in K \sim H$ there exists is $\in H^{\perp}$ such that ir $(x) \neq 1$.

Proof. Apply Lemma $2.1[19]$ to $K / H$.

Lemma 3.5. The Fourier transform of $T_{n l} f \in L^{1}(K / H)$ is the restriction of the Fourier transform $\hat{f}$ of $f$ to $H^{\perp}$.

Proof. Apply Theorem 2.4 and ([13], 5.1D).

REMARK 3.6. We just note the algebra homomorphism property of $T_{H}$ can be deduced from the above lemma using the uniqueness of Fourier transform for the special $K$ considered in this section.

REMARK 3.7. It is clear that from the proof of Theorem 3.2, that $\chi_{b}(K / H)=(K / H)^{\wedge}=H^{\perp}$.

LEMMA 3.8. If $H$ is compact or open then $H^{\perp}$ is open or compact respectively. 
Proof. If $H$ is compact then $\hat{H}$ is discrete. Since the restriction map $\lambda: \hat{K} \rightarrow \hat{H}([19],[10])$ is continuous and $H^{\perp}=\lambda^{-1}\{1\}$ we have that $H^{\lrcorner}$is open in $\hat{K}$. The other part follows using Theorem 3.2 since $K / H$ is discrete and therefore $(K / H)^{\wedge}$ is compact in this case.

Lemina 3.9. The space of $H$-invariant functions in $L^{1}(K)$ forms an ideal in $L^{1}(K)$.

Proof. Follows using ([9], 2.4) and ([13], 5.5A).

Lemma 3.10. $E \subset \hat{K}$ is a strong Ditkin (respectively, ultrastrong Ditkin) set if and only if there exists a net $\left\{\mu_{\alpha}\right\}_{\alpha \_\Lambda}$ of finite measures in $M(K)$ such that

(a) (i) for each $\alpha, \hat{\mu}_{\alpha}$ has compact support and equals 1 in a neighborhood of $E$.

(ii) for each $f \in k(E),\left\|\mu_{\alpha} * f\right\|_{1} \rightarrow 0$.

(b) There is a $C \geqq 0$ such that for all $f \in J(E)$ (in $\left.L^{1}(K)\right)$ with $\|f\|_{1} \leqq 1$ we have $\left\|f * \mu_{\alpha}\right\|_{1} \leqq C$ for all $\alpha$.

(Respectively, we have an $M \geqq 0$ with $\left\|\mu_{\alpha}\right\| \leqq M$ for all $\alpha$.)

Proof. Modify ([17], 2.2(b)).

THEOREM 3.11. Let $H$ be a compact subhypergroup of $K$ and $\Delta \subset H^{-}$. Then

(i) $\triangle$ is spectral for $L^{1}(K / H)$ if it is so for $L^{1}(K)$.

(ii) $\Delta$ is ultra-strong Ditkin (respectively, strong Ditlin, Calderon) for $L^{1}(K / H)$ if and only if it is so for $L^{1}(K)$.

(iii) $\Delta$ is sequentially strong Ditkin for $L^{1}(K / H)$ if it is so for $L^{1}(K)$. If $K$ is first countable and $\Delta$ is sequentially strong Ditkin for $L^{1}(K / H)$ then it is so for $L^{1}(K)$.

Proof. We shall only prove the ultra-strong Ditkin part of (ii) and indicate a proof for the strong Ditkin case. It will be clear how to formulate proof of the rest of the theorem.

Let $\Delta$ be ultra-strong Ditkin for $L^{1}(K)$. Then there is a net $\left\{f_{\alpha}\right\}_{\alpha \in d}$ in $L^{1}(K)$ such that for each $\alpha, \hat{f}_{\alpha}=0$ on a neighborhood $V_{\alpha}$ of $\Delta$ in $\hat{K}$ and $\hat{f}_{\alpha}$ has compact support; further $M=\sup \left\{\left\|f_{\alpha}\right\|_{1}: \alpha \epsilon\right.$ $1\}<\infty$ and for all $f \in L^{1}(K)$ with $\hat{f}=0$ on $\Delta$ we have $\left\|f_{\alpha} * f-f\right\|_{1} \rightarrow$ 0 . Let $\dot{f} \in L^{1}(K / H)$ be such that $\hat{\dot{f}}=0$ on $\Delta$. Then by Theorem 2.4 there exists $f$ in $L^{1}(K)$ such that $T_{H} f=\dot{f}$, so for $\gamma \in \Delta, \hat{f}(\gamma)=\hat{\dot{f}}(\gamma)=$ 0 by Lemma 3.5. Let $g_{\alpha}=T_{I I} f_{\alpha}(\alpha \in \Delta)$. Then by Lemma 3.5 again for each $\alpha, \hat{g}_{c}$ vanishes on the neighborhood $V_{\alpha} \cap H^{\perp}$ of $\Delta$ in $H^{\perp}$ 
and has compact support. Applying Theorem 2.4 we have that $\left\{g_{\alpha}\right\}$ serves as the required net.

Conversely, let $\Delta$ be ultra-strong Ditkin for $L^{1}(K / H)$, applying Lemma 3.10 there exists a net $\left\{\dot{\mu}_{\alpha}\right\}_{\alpha \in A}$ in $M(K / H)$ such that for each $\alpha, \hat{\dot{\mu}}_{\alpha}=1$ on a neighborhood $V_{\alpha}$ of $\Delta$ in $H^{\perp}$ and $\hat{\dot{\mu}}_{\alpha}$ has compact support, further $M=\sup \left\{\left\|\dot{\mu}_{\alpha}\right\|: \alpha \in \Lambda\right\}<\infty$ and for $\dot{f} \in L^{1}(K / H)$ with $\hat{\dot{f}}$ zero on $\Delta,\left\|\dot{f}_{*} \dot{\mu}_{\alpha}\right\|_{1} \rightarrow 0$. Let $\left\{u_{\beta}\right\}_{\beta \in B}$ be an approximate unit for $L^{1}(K)$ such that for each $\beta, \widehat{u}_{\beta}$ has compact support $U_{\beta}$ and $\left\|u_{\beta}\right\|_{1}=1$ ([9], 2.8). Let $W_{\beta}=U_{\beta} \cap H^{\perp}$. Then $W_{\beta}$ is compact so by ([9], 2.5) there exists $\dot{g}_{\beta} \in L^{1}(K / H)$ such that $\hat{\dot{g}}_{\beta}=1$ on $W_{\beta}$. Let $\dot{f}_{\alpha, \beta}=\dot{\mu}_{\alpha} * \dot{g}_{\beta}$. Then there exists $f_{\alpha, \beta} \in L^{1}(K)$ such that $T_{H} f_{\alpha, \beta}=\dot{f}_{\alpha, \beta}$ and $f_{\alpha, \beta}(x, \xi)=f_{\alpha, \beta}(x)$ for all $x \in K$ and $\xi \in H$. Let $D$ be the directed set $B \times \Lambda$. For $\rho=(\beta, \alpha) \in B \times \Lambda$, let $h_{\rho}=u_{\beta}-u_{\beta} * f_{\alpha, \beta}$ so that $\hat{h}_{\rho}=\widehat{u}_{\beta}\left(1-\hat{f}_{\alpha, \beta}\right)$ and therefore $\hat{h}_{\rho}$ is zero on $V_{\alpha}$ and has compact support contained in $U_{\beta}$. Now

$$
\left\|h_{\rho}\right\|_{1} \leqq\left\|u_{\beta}\right\|_{1}+\left\|u_{\beta} * f_{\alpha, \beta}\right\|_{1}=1+\left\|u_{\beta} * f_{\alpha, \beta}\right\|_{1} .
$$

To estimate $\left\|u_{\beta}^{*} f_{\alpha, \beta}\right\|_{1}$ we use $T_{I I} u_{\beta}^{*} \dot{g}_{\beta}=T_{I I} u_{\beta}$ which follows easily using uniqueness of Fourier transform and the fact that $\hat{\dot{g}}_{\beta}(\gamma)=1$ for $\gamma \in W_{\beta}$ and $\left(T_{H} u_{\beta}\right)^{\wedge}(\gamma)=0$ for $\gamma \in H^{\perp} \sim U_{\beta}=H^{\perp} \sim W_{\beta}$. This equality will be used in further computations as well. Now using Lemma 3.9 and Theorem 2.4 and ([13], 6.2B).

$$
\begin{aligned}
\left\|u_{\beta} * f_{a, \beta}\right\|_{1} & =\left\|T_{H}\left(u_{\beta} * f_{\alpha, \beta}\right)\right\|_{1}=\left\|T_{I I} u_{\beta} * T_{I I} f_{\alpha, \beta}\right\|_{1} \\
& =\left\|T_{I I} u_{\beta} * \dot{g}_{\beta} * \dot{\mu}_{\alpha}\right\|_{1} \\
& =\left\|T_{I I} u_{\beta} * \dot{\mu}_{\alpha}\right\|_{1} \leqq\left\|T_{I I} u_{\beta}\right\|_{1}\left\|\dot{\mu}_{\alpha}\right\| \leqq\left\|u_{\beta}\right\|_{1}\left\|\dot{\mu}_{\alpha}\right\| \leqq M .
\end{aligned}
$$

So $\left\|h_{\rho}\right\|_{1} \leqq 1+M$ for all $\rho$. Fix any $f \in L^{1}(K)$ such that $\hat{f}$ vanishes on $\Delta$. Then for each $n \in N$ there exists $\beta_{n} \in B$ such that $\| f-$ $f * u_{\beta} \|_{1}<1 / n$ for all $\beta \geqq \beta_{n}$. Also for all $n$ there exists $\alpha_{n}$ such that

$$
\left\|T_{I I} f * \dot{\mu}_{\alpha}\right\|_{1}<\frac{1}{n} \text { for all } \alpha \geqq \alpha_{n} .
$$

Let $\varepsilon>0$ be arbitrary. Let $n_{0} \in N$ be such that $1 / n_{0}<\varepsilon / 2$. Then for $\rho \geqq \rho_{0}=\left(\beta_{n_{0}}, \alpha_{n_{0}}\right)$

$$
\begin{gathered}
\left\|f * h_{\rho}-f\right\|_{1} \leqq\left\|f * u_{\beta}-f\right\|_{1}+\left\|f * u_{\beta} * f_{\alpha, \beta}\right\|_{1} \\
<\frac{1}{n_{0}}+\left\|f * u_{\beta} * f_{\alpha, \beta}\right\|_{1} .
\end{gathered}
$$

Further using Lemma 3.9 and Theorem 2.4 again 


$$
\begin{aligned}
\left\|f * u_{\beta} * f_{\alpha, \beta}\right\|_{1} & =\left\|T_{I I} f * T_{I I} u_{\beta} * \dot{g}_{\beta} * \dot{\mu}_{\alpha}\right\|_{1} \\
& =\left\|T_{I I} f * T_{I I} u_{\beta} * \dot{\mu}_{\alpha}\right\|_{1} \\
& \leqq\left\|T_{I I} u_{\beta}\right\|_{1}\left\|T_{I I} f * \dot{\mu}_{\alpha}\right\|_{1}<\frac{1}{n_{0}} .
\end{aligned}
$$

So $\left\|f * h_{\rho}-f\right\|_{1}<2 / n_{0}<\varepsilon$.

Hence $\left\{h_{\rho}\right\}_{\rho \in D}$ serves as a required net. Now we note that for $\dot{h} \in L^{1}(K / H)$.

$$
\begin{aligned}
\sup & \left\{\|\dot{h} * \dot{g}\|_{1}: \dot{g} \in J(\Delta) \text { in } L^{1}(K / H) \text { and }\|\dot{g}\|_{1} \leqq 1\right\} \\
& =\sup \left\{\left\|\dot{h} * T_{H}(\dot{g} \circ \pi)\right\|_{1}: \dot{g} \in J(\Delta) \text { in } L^{1}(K / H) \text { and }\|\dot{g}\|_{1} \leqq 1\right\} \\
& \leqq \sup \left\{\|\dot{h} \circ \pi * \dot{g} \circ \pi\|_{1}: \dot{g} \in J(\Delta) \text { in } L^{1}(K / H) \text { and }\|\dot{g}\|_{1} \leqq 1\right\} \\
& \leqq \sup \left\{\|\dot{h} \circ \pi * g\|_{1}: g \in J(\Delta) \text { in } L^{1}(K) \text { and }\|g\|_{1} \leqq 1\right\}
\end{aligned}
$$

So the proof for the ultra-strong Ditkin can be appropriately changed to show that if $\Delta$ is strong Ditkin for $L^{1}(K)$ then it is so for $L^{1}(K / H)$. For the converse part we choose $h_{\rho}$ as above using strong Ditkin version of Lemma 3.9 and note that for $f \in J(\Delta)$ in $L^{1}(K)$ with $\|f\|_{1} \leqq 1$ we have $T_{I I} f \in J(\Delta)$ (in $L^{1}(K / H)$ ) and $\left\|T_{I I} f\right\|_{1} \leqq$ 1 by Theorem 2.4 and Lemma 3.7; further we perform obvious computations to show that $\left\|f * h_{\rho}\right\|_{1} \leqq C$ for all $\rho$.

Corollary 3.12. Let $E \subset H^{\perp}$ and $\gamma \in Z(\hat{K})$. Then $E$ is Calderon (respectively, strong Ditkin, ultra-strong Ditkin) for $L^{1}(K / H)$ if and only if $\gamma E$ is so for $L^{1}(K)$.

Proof. It follows immediately from ([9], 3.7).

4. Quotient by subgroups of the center $Z(K)$. In this section $H$ will be a closed subgroup of the center $Z(K)$ of $K$ where $K$ is a commutative hypergroup such that its dual $\hat{K}$ is also a hypergroup under pointwise operations. As shown in ([9], 4.1) $K / H=\{x H: x \in$ $K\}$ is a commutative hypergroup with convolution defined by

$$
\int_{K / I I} f d p_{x I I} * p_{y H}=\int_{K} f \circ \pi d p_{x} * p_{y} .
$$

Let $\sigma$ be the Haar measure of $H$.

THeOREM 4.1. The Haar measure $\dot{m}$ on $K / H$ can be chosen so that

$$
\int_{K / I I} \int_{H} f(x \xi) d \sigma(\xi) d \dot{m}(\dot{x})=\int_{K} f d m \text { for all } f \in L^{1}(K) \text {. }
$$

Proof. Define $T_{I I}$ on $C_{00}(K)$ by $T_{I I} f(\dot{x})=\int_{I I} f(x \xi) d \sigma(\xi)$. Then 
proof for the group case ([16], III, 3.2, 4.7) or ([12], 28.54) can be modified to have the required result.

THEOREM 4.2. Let $T_{I I}$ be as in proof of Theorem 4.1.

(i) Every function $f$ in $L^{1}(K / H)$ has the form $T_{H} f$ for some $f \in L^{1}(K)$ with $\|\dot{f}\|_{1}=\|f\|_{1}$.

(ii) $T_{H}: L^{1}(K) \rightarrow L^{1}(K / H)$ is an algebra homomorphism with norm 1 .

Proof. (i) for $g \in C_{00}(K / H)$ select $f \in C_{00}(K)$ as in ([16], III, 4.2), (ii) involves simple computations.

THEOREM 4.3. For $\varphi \in H^{\perp}, \varphi^{\prime}(x H)=\varphi(x)$ defines an element of $(K / H)^{\wedge}, \varphi \rightarrow \Phi^{\prime}$ is a one-to-one map of $H^{\perp}$ onto $(K / H)^{\wedge}$. Hence $(K / H)^{\wedge}$ is also a hypergroup under pointwise operations.

Proof. ([12], 5.24(b)) can be modified using ([13], 10.3B, 3.2B) again to give an analogue of Lemma 2.3 in this case also. Hence the techniques of $([19], 4.4)$ can be used to have the required result.

Lemma 4.4. The Fourier transform of $T_{H} f \in L^{1}(K / H)$ is the restriction of the Fourier transform $\hat{f}$ of $f$ to $H^{\perp}$.

THEOREM 4.5. Let $\Delta \subset H^{\lrcorner}$. If $\Delta$ is spectral (respectively, Calderon, strong Ditkin, sequentially strong Ditkin, ultra-strong Ditkin) for $L^{1}(K)$ then $\Delta$ is so for $L^{1}(K / H)$.

Proof. The proof is similar to that of the corresponding parts of Theorem 3.11. We just note that analogues of other parts of Theorem 3.11 are not available since we do not have an ideal $I$ in $L^{1}(K)$ (corresponding to the ideal of invariant functions in $L^{1}(K)$ ) such that $T_{H}$ is an isometric algebraic homomorphism on $I$ onto $L^{1}(K / H)$.

5. Spectral synthesis in products of hypergroups. In [15] Reiter gave an example of a function $f$ in $L^{1}\left(\boldsymbol{R}^{n}\right)(n \geqq 3)$ and a $\psi$ in $L^{\infty}\left(\boldsymbol{R}^{n}\right)$ such that $\hat{f}=0$ exactly on the unit sphere $S^{n-1}$ in $\boldsymbol{R}^{n}$ and $\left\langle f_{y}, \psi\right\rangle \neq 0$ for some $y \in \boldsymbol{R}^{n}$ but $\left\langle(f * f)_{z}, \dot{\psi}\right\rangle=0$ for all $z \in \boldsymbol{R}^{n}$ which in turn gives that $f$ and $f * f$ generate different closed ideals in $L^{1}\left(\boldsymbol{R}^{n}\right)$ but have the same zero set $S^{n-1}$. Malliavim proved that every nondiscrete locally compact abelian group $\widehat{G}$ contains a nonspectral set for $A(\hat{G})$. It is well known that Malliavin's theorem is not true for hypergroups because every closed subset in $\hat{K}$ is even strong Ditkin for the hypergroup $K=Z_{+}$related to $p$-adic 
numbers ([11], [9] and [7]). However it was shown in [8] that not every closed subset of $Z_{+}^{*}$ is ultra-strong Ditkin thus showing that we have a weaker form of Malliavin's theorem for $Z_{+}$. In this section we first show that Malliavin's theorem fails even for $Z_{+} \times$ $K_{2}, K_{2}$ being a compact abelian group with countable dual and on the other hand we develop methods to find nonspectral and non ultra-strong Ditkin sets for hypergroups of the type $K=\boldsymbol{R}^{+} \times K_{2}$ or $K=Z_{+} \times K_{2}$ respectively, where $K_{2}$ is a locally compact hypergroup such that its dual $\hat{K}_{2}$ is a $\sigma$-compact hypergroup. We begin with a few lemmas. As in $([13], 10.5) K_{1} \times K_{2}$ can be made into a hypergroup in the following way, if $(s, t)$ and $(x, y)$ are in $K_{1} \times K_{2}$ then

$$
p_{(s, t)} * p_{(x, y)}=\left(p_{s} * p_{x}\right) \times\left(p_{t} * p_{y}\right),(s, t)^{\vee}=(\check{s}, \check{t})
$$

where $K_{1}, K_{2}$ are locally compact hypergroups.

Lemma 5.1. Let $K_{1}, K_{2}$ be locally compact commutative hypergroups. For every $\left(\chi_{1}, \chi_{2}\right) \in \widehat{K}_{1} \times \widehat{K}_{2}$, let $\left[\chi_{1}, \chi_{2}\right]$ denote the function $\left(x_{1}, x_{2}\right) \rightarrow \chi_{1}\left(x_{1}\right) \chi_{2}\left(x_{2}\right)$ defined on $K_{1} \times K_{2}$. Then the mapping $\theta:\left(\chi_{1}\right.$, $\left.\chi_{2}\right) \rightarrow\left[\chi_{1}, \chi_{2}\right]$ is a one-to-one map of $\hat{K}_{1} \times \hat{K}_{2}$ onto $\left(K_{1} \times K_{2}\right)^{\wedge}$. If $\hat{K}_{1}, \hat{K}_{2}$ are hypergroups then so is $\left(K_{1} \times K_{2}\right)^{\wedge}$.

LeMma 5.2. Let $K_{1}, K_{2}$ be commutative hypergroups such that $\hat{K}_{1}, \hat{K}_{2}$ are also hypergroups, $f_{1} \in L^{1}\left(K_{1}\right)$ and $f_{2} \in L^{1}\left(K_{2}\right)$. If $f(x, y)=$ $f_{1}(x) f_{2}(y)$ for $(x, y) \in K_{1} \times K_{2}$ then

(i) $\hat{f}\left(\left(\gamma_{1}, \gamma_{2}\right)\right)=\hat{f}_{1}\left(\gamma_{1}\right) \hat{f}_{2}\left(\gamma_{2}\right)$ for all $\left(\gamma_{1}, \gamma_{2}\right) \in \hat{K}_{1} \times \hat{K}_{2}$.

(ii) $f * f(x, y)=f_{1} * f_{1}(x) f_{2} * f_{2}(y)$.

Lemma 5.3. Let $K_{1}, K_{2}$ be as in Lemma 5.2, $f \in L^{1}\left(K_{1} \times K_{2}\right)$ and $\gamma_{2} \in \hat{K}_{2}$. There exists $f_{\gamma_{2}} \in L^{1}\left(K_{1}\right)$ such that $\hat{f}\left(\gamma_{1}, \gamma_{2}\right)=\hat{f}_{\gamma_{2}}\left(\gamma_{1}\right)$ for each $\gamma_{\perp} \in \hat{K}_{1}$.

Proof. Repeated applications of Fubini's theorem give that $f_{r_{2}}$ can be defined as $f_{\tau_{2}}\left(x_{1}\right)=\int_{K_{2}} f\left(x_{1}, x_{2}\right) \gamma_{2}\left(x_{2}\right) d m\left(x_{2}\right), m$ being the Haar measure of $K_{2}$.

The following results can be deduced from Theorem 3.11 and 4.5 above and ([9], 3.7 and 3.8).

Theorem 5.4. Let $K=K_{1} \times K_{2}, K_{1}$ and $K_{2}$ be as in Lemma 5.2 .

(i) If $K_{2}$ is compact or a locally compact abelian group then if $E \subset \hat{K}_{1}$ is not a spectral (respectively, Calderon sequentially 
strong Ditkin, ultra-strong Ditkin) set for $L^{1}\left(K_{1}\right)$ then $E \times\left\{1_{2}\right\}$ is not a spectral (respectively, Calderon, sequentially strong Ditkin, ultra-strong Ditkin) set for $L^{1}(K)$ where $1_{2}$ is the identity of $\hat{K}_{2}$.

(ii) If $K_{2}$ is compact, $\left(\gamma_{1}, \gamma_{2}\right) \in Z\left(\hat{K}_{1}\right) \times Z\left(\hat{K}_{2}\right)=Z\left(\hat{K}_{1} \times \widehat{K}_{2}\right)$ then $E \subset \hat{K}_{1}$ is Calderon (respectively, strong Ditkin, ultra-strong Ditkin) for $L^{1}\left(K_{1}\right)$ if and only if $\gamma_{1} E \times\left\{\gamma_{2}\right\}$ is Calderon (respectively strong Ditkin, ultra-strong Ditkin) for $L^{1}(K)$.

(iii) If $K_{2}$ is a compact abelian group with $\hat{K}_{2}$ countable, $\hat{K}_{1}$ is discrete except at a countable subset $S_{1}$ and every point of $S_{1}$ is Calderon (in particular when $S_{1} \subset Z\left(\hat{K}_{1}\right)$ ) then every closed subset of $\hat{K}_{1} \times \hat{K}_{2}$ is Calderon for $L^{1}(K)$.

REMARK 5.5. Theorem 5.4 (iii) gives us that Malliavin's theorem fails for more hypergroups such as $Z_{+} \times T^{n}, T$ being the circle group and $n$ any positive integer.

The basic lemmas of this section, ([9], 2.5) and the techniques of ([22], p. 419) can be further used to modify the proof of ([14], 3.8) and hence have the following generalizations to hypergroups for $p=1^{*}$ see also ([3], 2.5.5 (f)) ([1], p. 307) and ([2], p. 240).

THEOREM 5.6. Let $K_{1}, K_{2}$ be as in Lemma 5.2 with further assumption that $\hat{K}_{2}$ is $\sigma$-compact. If $E_{1} \subset \hat{K}_{1}$ is a nonspectral set, then $E_{1} \times \hat{K}_{2}$ is a nonspectral set for $L^{1}\left(K_{1} \times K_{2}\right)$.

THEOREM 5.7. Let $K=\boldsymbol{R}^{n} \times K_{2}(n \geqq 3)$ where $K_{2}$ is such that $\hat{K}_{2}$ is a $\sigma$-compact hypergroup and let $E$ be a nonempty closed open subset of $\hat{K}_{2}$. Then there is an $f \in L^{1}(K)$ such that the closed ideal generated by $f * f$ does not contain $f$ and $Z(\hat{f})=S^{n-1} \times E$.

Proof. Let $f_{1}$ in $L^{1}\left(\boldsymbol{R}^{n}\right)$ and $\psi_{1}$ in $L^{\infty}\left(\boldsymbol{R}^{n}\right)$ be the same as $f, \psi$ in ([15], $\S 2$, Theorem 1) and $g_{1}$ be a rotationally invariant function in $L^{1}\left(\boldsymbol{R}^{n}\right)$ such that $\hat{g}_{1}>0$. Using ([9], 2.5) and techniques of ([22], p. 419) there are $f_{2}, g_{2} \in L^{1}\left(K_{2}\right)$ such that $Z\left(\hat{g}_{2}\right)=E, Z\left(\hat{f}_{2}\right)=\hat{K}_{2} \sim E$ and $\widehat{g}_{2} \geqq 0, \hat{f}_{2} \geqq 0$. Since $E \neq \Phi$ and the zero set of $I_{g_{2}}$, the closed ideal in $L^{1}\left(K_{2}\right)$ generated by $g_{2}$ is $E$, we have that $f_{2} \notin I_{g_{2}}$. So there exists $\psi_{2} \in L^{\infty}\left(K_{2}\right)$ such that $\left\langle f_{2}, \psi_{2}\right\rangle \neq 0$ but $\left\langle h, \psi_{2}\right\rangle=0$ for all $h \in I_{g_{2}}$. Define $f$ on $\boldsymbol{R}^{n} \times K_{2}$ by

$$
f\left(x_{1}, x_{2}\right)=f_{1}\left(x_{1}\right) f_{2}\left(x_{2}\right)+g_{1}\left(x_{1}\right) g_{2}\left(x_{2}\right) .
$$

Let $\psi$ be defined on $\boldsymbol{R}^{n} \times K_{2}$ by $\psi\left(x_{1}, x_{2}\right)=\psi_{1}\left(x_{1}\right) \psi_{2}\left(x_{2}\right)$. Then $\psi \epsilon$ $L^{\infty}\left(\boldsymbol{R}^{n} \times K_{2}\right)$. Clearly $S^{n-1} \times E$ is the zero set of $\hat{f}$. Consider $\left(y_{1}\right.$,

* For $p$-spectral synthesis in hypergroups see [23] and [24]. 
$\left.y_{2}\right) \in \boldsymbol{R}^{n} \times K_{2}$ where $y_{2}$ is the identity of $K_{2}$ and $y_{1}$ is an element of $\boldsymbol{R}^{n}$ with the first coordinate between 0 and $1 / 2$ and with other coordinates zero. Then $f_{y}=\left(f_{1}\right)_{y_{1}} f_{2}+\left(g_{1}\right)_{y_{1}} g_{2}$. Since $\left\langle f_{2}, \psi_{2}\right\rangle \neq 0$ and $\left\langle g_{2}, \psi_{2}\right\rangle=0$, Reiter's computations in ([15], $\S 2$, Theorem 1) can be modified to give that $\left\langle f_{y}, \psi\right\rangle \neq 0$. But for any $z=\left(z_{1}, z_{2}\right) \in \boldsymbol{R}^{n} \times K_{2}$ using Lemma 5.2 and ([9], 2.2, 2.4),

$$
\begin{aligned}
& \left\langle(f * f)_{z}, \psi\right\rangle=\left\langle\left(f_{1} * f_{1}\right)_{z_{1}}, \psi_{1}\right\rangle\left\langle\left(f_{2} * f_{2}\right)_{z_{2}}, \psi_{2}\right\rangle \\
& +2\left\langle\left(f_{1} * g_{1}\right)_{z_{1}}, \psi_{1}\right\rangle\left\langle\left(f_{2} * g_{2}\right)_{z_{2}}, \psi_{2}\right\rangle \\
& +\left\langle\left(g_{1} * g_{1}\right)_{z_{1}}, \psi_{1}\right\rangle\left\langle\left(g_{2} * g_{2}\right)_{z_{2}}, \psi_{2}\right\rangle=0,
\end{aligned}
$$

because $\left\langle h, \psi_{2}\right\rangle=0$ for all $h$ in $I_{g_{2}}, I_{g_{2}}$ contains $\left(f_{2} * g_{2}\right)_{z_{2}}=\left(f_{2}\right)_{z_{2}} * g_{2}$ and $\left(g_{2} * g_{2}\right)_{z_{2}}=\left(g_{2}\right)_{z_{2}} * g_{2}$ using ([9], 2.4, 2.11) and $\left\langle\left(f_{1} * f_{1}\right)_{z_{1}}, \psi_{1}\right\rangle$ is zero. Hence $f$ and $f * f$ generate different closed ideals in $L^{1}\left(\boldsymbol{R}^{n} \times K_{2}\right)$.

The proof of ([15], $\S 2$, Theorem 2) can be used in the same way to obtain the following generalization of the above theorem.

THEOREM 5.8. If $T$ is an arbitrary closed set in $\boldsymbol{R}^{n}$ such that $S^{n-1} \sim T \neq \Phi$ and $E$ is as in Theorem 5.7, then for $n \geqq 3$ there exists $f$ in $L^{1}\left(\boldsymbol{R}^{n} \times K_{2}\right)$ whose zero set is $\left(S^{n-1} \cup T\right) \times E$ and $f, f * f$ generate different closed ideals in $L^{1}\left(\boldsymbol{R}^{n} \times K_{2}\right)$.

THeOREM 5.9. Let $K_{2}$ be as in Theorem 5.7. For $n \geqq 3\left(S^{n-1} \cup\right.$ $T) \times E$ is a nonspectral set in $\boldsymbol{R}^{n} \times \hat{K}_{2}$ for $L^{1}\left(\boldsymbol{R}^{n} \times K_{2}\right)$ where $E$ is a nonempty closed open set in $\hat{K}_{2}$ and $T$ is as in Theorem 5.8. In particular $S^{n-1} \times E$ is nonspectral.

Proof. Follows from Theorem 5.8.

Now we consider $\boldsymbol{R}^{+}=[0, \infty)$ viewed as the hypergroup $\boldsymbol{R}^{+}=$ $G_{B}$ of $B$-orbits in $G=\boldsymbol{R}^{n}(n \geqq 3)$ where $B$ is the compact group of rotations in $G$. See the discussion in ([18], §3) and ([9], 4.3).

THEOREM 5.10. $\left\{x_{0}\right\} \times E$ is a nonspectral set in $\boldsymbol{R}^{+} \times \hat{K}_{2}$ where $x_{0} \neq 0$ is any point of $\boldsymbol{R}^{+}$and $K_{2}$ and $E$ are as in Theorem 5.7.

Proof. It follows immediately from Theorem 5.7 since $f_{1}$ and $g_{1}$ are rotationally invariant.

REMARK 5.11. In the above theorems $E$ can be a subset of $\chi_{b}\left(K_{2}\right)$ if we assume the regularity of $L^{1}\left(K_{2}\right)$ on $\chi_{b}\left(K_{2}\right)$, the hypergroup $\boldsymbol{R}^{+}$can be replaced by a hypergroup $K_{1}$ whose dual $\hat{K}_{1}$ is a $\sigma$-compact hypergroup and the set $\left\{x_{0}\right\}$ by a set $E_{1}$ in $\hat{K}_{1}$ if there exists a function $f_{1}$ in $L^{1}\left(K_{1}\right)$ such that $f_{1}$ and $f_{1} * f_{1}$ generate 
different closed ideals in $L^{1}\left(K_{1}\right)$ and the zero set of $\hat{f}_{1}$ is exactly $E_{1}$. Now we discuss another method to obtain nonspectral sets for certain hypergroups whose obvious generalizations can be easily formulated in view of discussion in ([9], 4.3).

THEOREM 5.12. Let $K=K_{1} \times K_{2}$ where $K_{2}$ is as in Theorem 5.7 and $K_{1}$ is the dual $\hat{F}$ of the hypergroup $F$ of conjugacy classes of the compact group $S U(2)$ ([13], 15.4) and ([9], 4.7). Then $\left\{\chi_{0}\right\} \times$ $E$ is a nonspectral set in $\hat{K}_{1} \times \hat{K}_{2}$ where $\theta \in(0, \pi)$ and $E$ is as in Theorem 5.7.

Proof. Let $f_{2}, g_{2} \in L^{1}\left(K_{2}\right)$ be as in Theorem 5.7 and $g_{1} \in L^{1}\left(K_{1}\right)$ be such that $\hat{g}_{1}>0$. Let $m(\{n\})=\alpha_{n}$ for $n \in K_{1}$ so that $\alpha_{n}>0$ for all $n \in K_{1}$. For $\theta \in(0, \pi)$ let $g_{\theta}$ be as in ([9], 4.7). Then $g_{\theta} \in L^{\infty}\left(K_{1}\right)$. For a function $h$ on $K$ vanishing outside $\{0,1,2\}$,

$$
\hat{h}\left(\chi_{0}\right)=h(0) \alpha_{0}+h(1) \alpha_{1} \cos \theta+h(2) \alpha_{2}\left(1-\frac{4}{3} \sin ^{2} \theta\right)
$$

and $\left\langle h, g_{0}\right\rangle=-h(1) \alpha_{1} \sin \theta-(8 / 3) h(2) \alpha_{2} \sin \theta \cos \theta$. Taking $f_{1}(0) \alpha_{0}=$ $\sin 2 \theta, f_{1}(1) \alpha_{1}=-2 \sin \theta, f_{1}(2) \alpha_{2}=0$ we have $\hat{f}_{1}\left(\chi_{\theta}\right)=0$ but $\left\langle f_{1}, g_{0}\right\rangle \neq$ 0 and $\hat{f}_{1}\left(\chi_{0^{\prime}}\right)=2 \sin \theta\left(\cos \theta-\cos \theta^{\prime}\right) \neq 0$ for $\theta \neq \theta^{\prime}$. Also taking

$$
\begin{aligned}
& \varphi_{1}(0) \alpha_{0}=\frac{1}{3}+\frac{4}{3} \cos ^{2} \theta, \varphi_{1}(1) \alpha_{1}=-\frac{8}{3} \cos \theta \text { and } \\
& \varphi_{1}(2) \alpha_{2}=1, \text { we have } \hat{\varphi}_{1}\left(\chi_{\theta}\right)=0=\left\langle\varphi_{1}, g_{\theta}\right\rangle
\end{aligned}
$$

but $\hat{\varphi}_{1}\left(\chi_{\theta^{\prime}}\right)=4 / 3\left(\cos \theta-\cos \theta^{\prime}\right)^{2} \neq 0$ for $\theta \neq \theta^{\prime}$. Let

$$
f(x, y)=f_{1}(x) f_{2}(y)+g_{1}(x) g_{2}(y)
$$

and

$$
\varphi(x, y)=\varphi_{1}(x) f_{2}(y)+g_{1}(x) g_{2}(y) .
$$

Clearly the zero set of $\hat{f}$ as well as that of $\hat{\varphi}$ is $\left\{\chi_{\theta}\right\} \times E$. Define $\chi_{\theta} \gamma_{2}(x, y)=\chi_{\theta}(x) \gamma_{2}(y)$ and $g_{0} \gamma_{2}(x, y)=g_{\theta}(x) \gamma_{2}(y)$ and let $E_{\theta}=\left\{\chi_{\theta} \gamma_{2}: \gamma_{2} \in\right.$ $E\}$ and $F_{\theta}=\left\{g_{\theta} \gamma_{2}: \gamma_{2} \in E\right\}$. Then in view of discussion in ([9], 4.3 and 4.7) $I=\left\{h:\langle h, \psi\rangle=0, \psi \in E_{\theta}\right\}$ and $J=\left\{h:\langle h, \psi\rangle=0, \psi \in E_{\theta} \cup\right.$ $F_{\theta}$ \} are distinct closed ideals with the same zero set $E_{\theta}$ since $\varphi \in J$ and $f \in I \sim J$. Hence $\left\{\chi_{\theta}\right\} \times E$ is a nonspectral set for $L^{1}\left(K_{1} \times K_{2}\right)$. In view of ([9], 4.3 and 4.7) the same conclusion also follows when we take $\varphi(x, y)=\left(f_{1} * f_{1}\right)(x) f_{2}(y)+g_{1}(x) g_{2}(y)$.

REMARK 5.13. Let $K_{1}=[0, \infty)$ be the hypergroup named as Naimark's example $([13], 9.5)([9], 4.8)$ and $K_{2}, E$ be as in Theorem 5.7. Fix any $b>0$ and let $a=b^{2}$ then $\left\{\chi_{a}\right\} \times E$ is a nonspectral 
set for $L^{1}\left(K_{1} \times K_{2}\right)$. Let $f_{2}$ and $g_{2}$ be as in Theorem 5.7 and for any fixed $c>1$ take $g_{1}(x)=f_{c}(x)=e^{-c x} / \sinh x$ ([13], 9.5), we have $\hat{g}_{1}>0$. Select $f_{1}$ and $\varphi_{1}$ in $L^{1}\left(K_{1}\right)$ as follows

$$
\begin{aligned}
f_{1}(x) & =\frac{1}{\sinh x} \text { for } 0<x \leqq \frac{2 \pi}{b} \\
& =0 \quad \text { otherwise }, \\
\mathcal{P}_{1}(x) & =\frac{1-b x}{\sinh x} \text { for } 0<x \leqq \frac{\pi}{2 b} \\
& =0 \quad \text { otherwise }
\end{aligned}
$$

and define

$$
\begin{aligned}
& f(x, y)=f_{1}(x) f_{2}(y)+g_{1}(x) g_{2}(y) \\
& \mathcal{P}(x, y)=\varphi_{1}(x) f_{2}(y)+g_{1}(x) g_{2}(y) .
\end{aligned}
$$

The zero set of $\hat{f}$ and $\hat{\varphi}$ is $\left\{\chi_{a}\right\} \times E$. Let

$$
i_{1}(x)=\frac{x \cos b x}{b \sinh x} \text { for all } x \in K .
$$

For $\gamma_{2} \in E$ let $\psi(x, y)=\psi_{1}(x) \gamma_{2}(y)$. Then $\psi^{\prime} \in L^{\infty}\left(K_{1} \times K_{2}\right)$. Also $\left\langle f_{1}, \psi_{1}\right\rangle=0$ and $\left\langle\varphi_{1}, \psi_{1}\right\rangle \neq 0$ and therefore $\langle f, \dot{\psi}\rangle=0$ and $\langle\varphi, \psi\rangle \neq$ 0 . As argued in Theorem 5.12 in view of discussion in ([9], 4.8) we have that $f$ and $\varphi$ generate different closed ideals.

REMARK 5.14. Let $K_{1}$ be $\boldsymbol{R}^{+}, \hat{F}$ or Naimark's example and $K_{2}$ be the hypergroup $Z_{+}\left([11]\right.$, [8]). Then $\hat{K}_{2}$ is a $\sigma$-compact hypergroup. If $E_{2}$ is a closed set in $\hat{K}_{2}$, which is finite and does not contain the identity or is the complement of a finite set (and therefore contains the identity) then $E_{2}$ is open. Even though by ([8], Example 10) it is ultra-strong Ditkin for $L^{1}\left(K_{2}\right)$, the above results can be applied to have that $\{\gamma\} \times E_{2}$ is nonspectral for $L^{1}\left(K_{1} \times K_{2}\right)$ where $\gamma \in \hat{K}_{1} \sim Z\left(\hat{K}_{1}\right)$. Our next result further shows that if $E_{2}$ is an infinite closed set in $Z_{*}^{*}$ which is not open (and hence has an infinite complement and therefore by ([8], Example 10) it is not ultra-strong Ditkin for $L^{1}\left(K_{2}\right)$ ), then $\{\gamma\} \times E_{2}$ is not ultra-strong Ditkin for $L^{1}\left(K_{1} \times K_{2}\right)$.

THEOREM 5.15. Let $K=Z_{+} \times K_{2}$, where $K_{2}$ is as in Lemma 5.2 and $Z_{+}$is the hypergroup considered in ([11], [8]). Then $E_{1} \times$ $E_{2}$ is a non-ultra-strong Ditkin set for $L^{1}(K)$ where $E_{1}$ is (as in [8]) an infinite closed subset of $Z_{+}^{*}$ such that its complement is also infinite and $E_{2}$ is a nonempty closed subset of $\hat{K}_{2}$.

Proof. Let $\mathcal{P}=\hat{g}_{1}$ in $A\left(Z_{+}^{*}\right)$ be as in ([8], Example 10(ii)(c)). 
Also there exists $g_{2} \in L^{1}\left(K_{2}\right)$ such that $\hat{g}_{2} \neq 0$ on $E_{2}$. Define $f$ on $Z_{+} \times K_{2}$ by $f(x, y)=g_{1}(x) g_{2}(y)$. Clearly $f$ belongs to $k\left(E_{1} \times E_{2}\right)$. Suppose $E_{1} \times E_{2}$ is an ultra-strong Ditkin set then as in [8] the Banach algebra $k\left(E_{1} \times E_{2}\right)$ has a bounded approximate identity and therefore it has factorization by Cohen's factorization theorem. So there exist $g, h \in k\left(E_{1} \times E_{2}\right)$ such that $f=g * h$. Fix $\gamma_{2} \in E_{2}$ such that $\hat{g}_{2}\left(\gamma_{2}\right) \neq 0$ and without loss of generality we may take $\hat{g}_{2}\left(\gamma_{2}\right)=1$. So for all $\gamma_{1} \in Z_{+}^{*}$ using, Lemma 5.3,

$$
\begin{aligned}
\hat{g}_{1}\left(\gamma_{1}\right) & =\hat{f}\left(\gamma_{1}, \gamma_{2}\right)=\hat{g}\left(\gamma_{1}, \gamma_{2}\right) \hat{h}\left(\gamma_{1}, \gamma_{2}\right) \\
& =\hat{g}_{\gamma_{2}}\left(\gamma_{1}\right) \hat{h}_{\gamma_{2}}\left(\gamma_{1}\right)
\end{aligned}
$$

that is $g_{1}=g_{\gamma_{2}} * h_{\gamma_{2}}$. Since $g, h \in k\left(E_{1} \times E_{2}\right), \quad g_{\gamma_{2}}$ and $h_{\gamma_{2}}$ belongs to $k\left(E_{1}\right)$ which is a contradiction to the proof of ([8], Example 10(ii)(c)). Hence $E_{1} \times E_{2}$ is a non ultra-strong Ditkin set for $L^{1}\left(Z_{+} \times K_{2}\right)$.

We thank the referee for his comments and suggestions.

\section{REFERENCES}

1. A. Atzmon, Non-singly generated closed ideals in group algebras, Israel J. Math., 7 (1969), 303-310.

2. — Non-finitely generated closed ideals in group algebras, J. Functional Analysis, 11 (1972), 231-249.

3. J. Benedetto, Spectral synthesis, B. G. Teubner, Stuttgart, 1975.

4. W. R. Bloom, Sets of p-spectral synthesis, Pacific J. Math., 66 (1975), 7-19.

5. F. Bruhat, Sur les representations induites des groupes de Lie, Bull. Soc. Math. France, 84 (1956), 97-205.

6. A. K. Chilana, Harmonic analysis and hypergroups, Proceedings of the symposium on recent developments in Mathematics, Allahabad, (1978), 93-121.

7. A. K. Chilana and A. Kumar, Spectral synthesis in Segal algebras on hypergroups, Pacific J. Math., 80 (1979), 59-76.

8. - Ultra-strong Ditkin sets in hypergroups, Proc. Amer. Math. Soc., 77 (1979), 353-358.

9. A. K. Chilana and K. A. Ross, Spectral synthesis in hypergroups, Pacific J. Math., 76 (1978), 313-328.

10. C. F. Dunkel, The measure algebra of a locally compact hypergroup, Trans. Amer. Math. Soc., 179 (1973), 331-348.

11. C. F. Dunkel and D. E. Ramirez, A family of countable $P^{*}$-hypergroups, Trans. Amer. Math. Soc., 202 (1975), 339-356.

12. E. Hewitt and K. A. Ross, Abstract Harmonic Analysis $I$ and II, Springer-Verlag, 1963 and 1970.

13. R. I. Jewett, Spaces with an abstract convolution of measures, Advances in Math., 18 (1975), 1-101.

14. G. W. Mackey, Induced representations of locally compact groups, Ann. of Math., $\mathbf{5 5}$ (1972), 101-139.

15. H. Reiter, Contribution to harmonic analysis IV, Math. Ann., 133 (1958), 467-476.

16. Classical Harmonic Analysis and Locally Compact Groups, Oxford Math. Monograph, 1968.

17. H. P. Rosenthal, On the existence of approximate identities in ideals of group algebras, Ark. Mat., 7 (1969), 185-191. 
18. K. A. Ross, Hypergroups and centers of measures of algebras, Symp. Math., XXII (1977), 189-203.

19. , Centers of hypergroups, Trans. Amer. Math. Soc., 243 (1978), 251-269.

20. R. Spector, Apercu de la theorie des hypergroupes, Lecture notes on Mathematics, 497 (Analyse Harmonique sur les Groupes de Lie, Sem. Nancy-Strusburg 1973-1975, Springer-Verlag).

21. _- Mesures invariantes sur les hypergroupes, Trans. Amer. Math. Soc., 239 (1978), 147-165.

22. C. R. Warner, Closed ideals in the group algebra $L^{1} \backslash L^{2}(G)$, Trans. Amer. Math. Soc., 121 (1966), 408-423.

23. A. Kumar, Doctoral dissertation, Delhi University (1980).

24. A. Kumar and A. I. Singh, p-spectral synthesis in hypergroups, (communicated)

Received May 4, 1979 and in revised form December 5, 1979.

UNIVERSiTy OF DeLHi

Delhi, 110007 India 


\section{PACIFIC JOURNAL OF MATHEMATICS}

\section{EDITORS}

DONALD BABBITT (Managing Editor)

University of Galifornia

Los Angeles, California 90024

Hugo RossI

University of Utah

Salt Lake City, UT 84112

C. C. MOORE AND ANDREW OGG

University of California

Berkeley, CA 94720

\section{J. DugundjI}

Department of Mathematics University of Southern California

Los Angeles, California 90007

R. Finn AND J. Milgram

Stanford University

Stanford, California 94305

\section{ASSOCIATE EDITORS}

R. ARENS

E. F. BECKENBACH

B. H. NeUmanN

F. WOLF

K. YosHIDA

\section{SUPPORTING INSTITUTIONS}

UNIVERSITY OF ARIZONA

UNIVERSITY OF BRITISH COLUMBIA

CALIFORNIA INSTITUTE OF TECHNOLOGY

UNIVERSITY OF CALIFORNIA

MONTANA STATE UNIVERSITY

UNIVERSITY OF NEVADA, RENO

NEW MEXICO STATE UNIVERSITY

OREGON STATE UNIVERSITY
UNIVERSITY OF OREGON

UNIVERSITY OF SOUTHERN CALIFONIA

STANFORD UNIVERSITY

UNIVERSITY OF HAWAII

UNIVERSITY OF TOKYO

UNIVERSITY OF UTAH

WASHINGTON STATE UNIVERSITY

UNIVERSITY OF WASHINGTON 


\section{Pacific Journal of Mathematics}

\section{Vol. 94, No. 1 \\ May, 1981}

Willy Brandal, Conditions for being an FGC domain $\ldots \ldots \ldots \ldots \ldots \ldots \ldots$

Allan Calder and Frank Williams, Incompressibility of maps and the

homotopy invariance of Čech cohomology $\ldots \ldots \ldots \ldots \ldots \ldots \ldots \ldots \ldots$

Jacques Chaumat, Quelques propriétés du couple d'espaces vectoriels

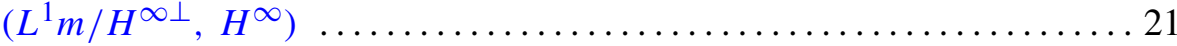

Manfred Droste and Rüdiger Göbel, Products of conjugate permutations . . 47

Jean Esterle, Rates of decrease of sequences of powers in commutative

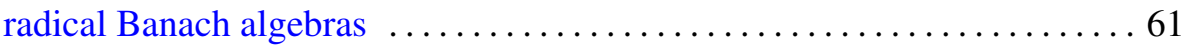

Allan Fryant, Ultraspherical expansions and pseudo analytic functions . . . 83

John Hannah, Homogenization of regular rings of bounded index. II . . . . . 107

Shigeru Haruki, On the theorem of S. Kakutani-M. Nagumo and J. L. Walsh

for the mean value property of harmonic and complex polynomials $\ldots .113$

Hugh M. Hilden, Representations of homology 3-spheres $\ldots \ldots \ldots \ldots \ldots 125$

Craig Huneke, A characterization of locally Macaulay completions . . . . . 131

Takesi Isiwata, Closed ultrafilters and realcompactness ................. 139

Joseph Weston Kitchen, Jr. and David A. Robbins, Tensor products of

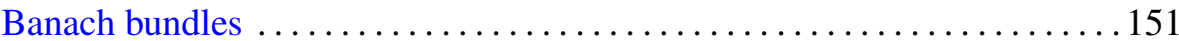

Allan J. Kroopnick, Note on bounded $L^{p}$-solutions of a generalized Liénard

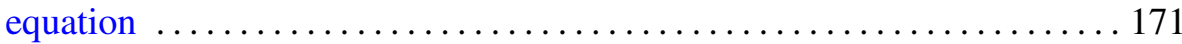

Ajay Kumar and Ajit Kaur Chilana, Spectral synthesis in products and quotients of hypergroups

Charles Livingston, Homology cobordisms of 3-manifolds, knot

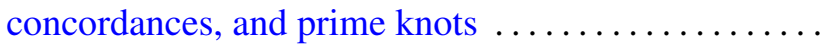

Hans Opolka, Projective representations of finite groups in cyclotomic fields

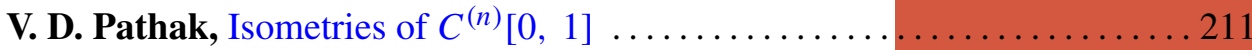

Mark Allan Pinsky, On the spectrum of Cartan-Hadamard manifolds . . . . . 2223

Judith Roitman, The number of automorphisms of an atomic Boolean

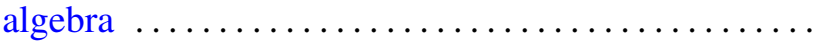

Kai Wang, Locally smooth torus group actions on integral cohomology complex projective spaces 